\title{
Drugs, guns and cars: how far we have come to improve safety in the United States; yet we still have far to go
}

James Dodington ${ }^{1-4}$, Pina Violano ${ }^{3,4}$, Carl R. Baum ${ }^{1,2}$ and Kirsten Bechtel ${ }^{1-4}$

Significant breakthroughs in the field of injury prevention and childhood safety have occurred during the past half-century. For example, the Poison Prevention Packaging Act of 1970 and the institution of child passenger safety laws are responsible for a significant reduction in injuries among children and adolescents. This review will focus on the following three topics because of their significant contribution to pediatric injury morbidity, especially among adolescents, and their promise for further effective prevention research. Opioid overdoses by adolescents and young adults are increasing; however, the use of naloxone by bystanders represents a life-saving development in opioid overdose prevention that deserves further investigation. Youth firearm injury remains a major cause of death and disability in adolescents. Despite a lack of robust injury prevention research on the topic, the development of novel approaches to access and examine firearm injury data is leading to exploration of public health approaches to reduce these injuries. Finally, despite legislative and educational efforts surrounding child passenger safety and graduated driver license laws, motor vehicle crashes are still a leading cause of injury for both children and adolescents; however, research on these laws holds the opportunity for significant reduction in injuries. Focused efforts to reduce unintentional injuries from opiate overdoses, firearms and motor vehicle crashes may produce a breakthrough in the field of injury prevention similar to that of the Poison Prevention Packaging Act.

I: $\mathrm{n}$ the past half-century, significant advances in injury prevention research have been able to identify measures that can prevent premature death or disability for youth. According to the Centers for Disease Control and Prevention, injuries, by both unintentional and intentional means, account for the leading causes of death and disability for those between the ages of 1 and $25 \mathrm{y}(1)$. These injuries are often seen as especially important in public health efforts, given that they represent a critical loss in a population that could otherwise go on to contribute to society for decades. In addition, the interventions put forth to prevent injuries are often surprisingly simple and yet can have widespread and long-lasting impact. A key example of this type of intervention was the Poison Prevention Packaging Act of 1970. In the decade that followed this legislation, it was estimated that nearly 200,000 pediatric unintentional ingestions were prevented (2).

This review will focus on opioid overdoses, firearm injury and motor vehicle crashes because of their disproportionate contribution to injury related fatalities for children and adolescents in the United States (Table 1). Notably, deaths by these three mechanisms account for the majority of injury fatalities in the age group less than 25 , and require further research and efforts around injury prevention (Table 1). In addition, the trend in the number of injury fatalities for these mechanisms in the 15-24 age group has remained high and relatively stable after modest improvement over the past $10 \mathrm{y}$, which makes the case that further research is needed to combat these leading causes of death (Figure 1). The possible mechanisms to be explored around injury prevention are vast, as are the interventions to reduce injury. Given this fact, this review will examine the historical perspective of each topic chosen, with a focus on key research publications, and then examine one intervention for each mechanism that represents an area yet to be fully explored that holds significant promise for further research.

\section{OPIOID OVERDOSES}

Since 2013, unintentional poisonings have become the 10th leading cause of injuries treated in US emergency departments in the 15-24-y-old age group (1). During this same time period, the misuse of prescription drugs, such as opioid medications, has also become a significant problem amongst this age group in the United States. The National Survey on Drug Use and Health (NSDUH) demonstrated that about 15 million people aged $12 \mathrm{y}$ or older misused prescription drugs in 2014; of these prescription drugs, opioids were misused more often than any other drug, except ethanol and marijuana (3). The increasing prevalence of prescription opioid misuse is due in part to the increasing availability of these drugs within the general population, and the perception amongst adolescents and young adults that because these drugs are prescribed, they are safe, whether used correctly or not (4). In addition to misuse of prescription opioids, the use of heroin has also increased over the past decade. In 2013, an estimated 169,000 individuals aged 12 or older used heroin for the first time in the previous year; of these, 21,000 were adolescents (12-18 y old) (5).

\footnotetext{
'Department of Pediatrics, Yale School of Medicine, New Haven, Connecticut; ${ }^{2}$ Department of Emergency Medicine, Yale School of Medicine, New Haven, Connecticut; ${ }^{3}$ Department of Injury Prevention, Community Outreach and Research, Yale-New Haven Children's Hospital, New Haven, Connecticut; ${ }^{4}$ Injury Free Coalition for Kids of New Haven, New Haven, Connecticut. Correspondence: Kirsten Bechtel (Kirsten.bechtel@yale.edu)

Received 1 May 2016; accepted 13 September 2016; advance online publication 26 October 2016. doi:10.1038/pr.2016.193
} 
Table 1. 2014-leading causes of injury deaths for children and young adults ages $0-24$

\begin{tabular}{|c|c|c|c|c|c|}
\hline \multirow[b]{2}{*}{ Rank } & \multicolumn{5}{|c|}{ Age groups } \\
\hline & $<1$ & $1-4$ & $5-9$ & $10-14$ & $15-24$ \\
\hline 1 & Unintentional Suffocation (991) & Unintentional drowning (388) & MV traffic (345) & MV traffic (384) & MV traffic $(6,531)^{a}$ \\
\hline 3 & $\begin{array}{l}\text { Homicide Unspecified, } \\
\text { classifiable (83) }\end{array}$ & Homicide unspecified (149) & Unintentional fire/burn (68) & Suicide firearm (174) & $\begin{array}{l}\text { Unintentional } \\
\text { poisoning }(3,492)^{\mathrm{a}}\end{array}$ \\
\hline
\end{tabular}

Data highlighting increase in case fatalities ( $n$ ) for the Age Group 15-24, for the three mechanism discussed within this review.

Source: National Center for Health Statistics (NCHS), National Vital Statistics System.

National Center for Injury Prevention and Control, CDC, WISQARS (1)

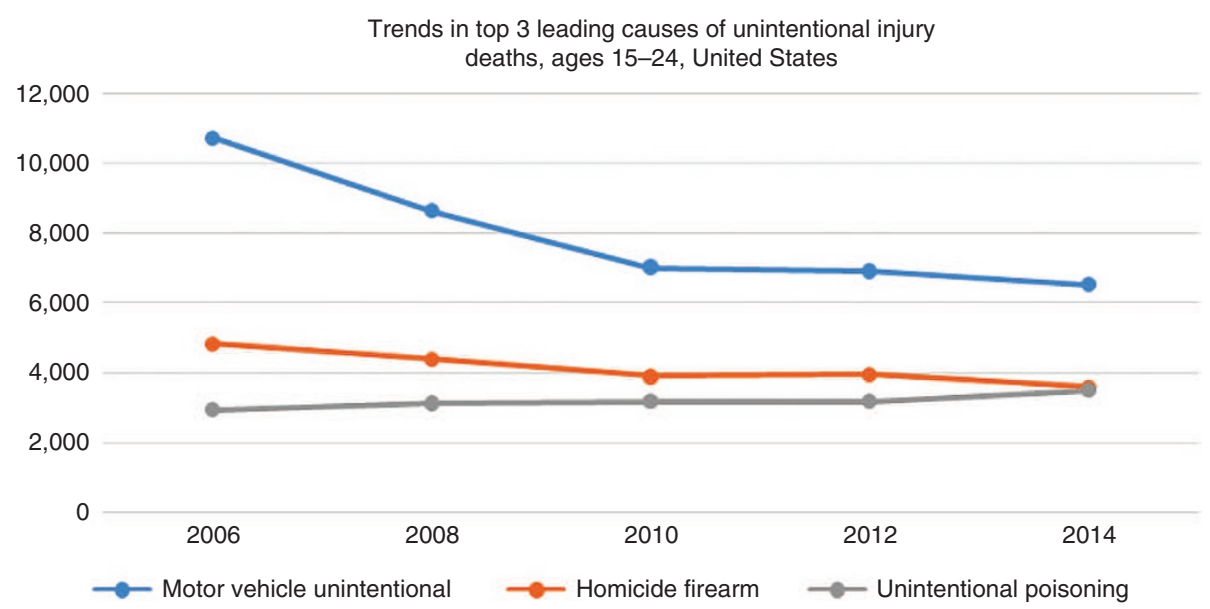

Figure 1. Trends in top 3 leading causes of unintentional injury deaths, ages 15-24, United States. Data Source: National Center for Health Statistics (NCHS), National Vital Statistics System. National Center for Injury Prevention and Control, CDC, WISQARS (1).

The rise in prescription drug misuse and heroin use is paralleled by a nearly fourfold increase in opioid overdose deaths during the past decade (6). In 2014, drug overdoses caused more deaths in the United States than during any previous year on record (7). To put this alarming trend in perspective, there were more drug overdose deaths in the United States than deaths from motor vehicle crashes in 2014; of these overdose deaths, 3,798 were among adolescents and young adults $15-24$ y old (8).

At present, there are several multidisciplinary approaches to reduce opioid-related overdoses and deaths among adolescents and young adults. These include programs that reduce the risk of misuse through safe storage and disposal of prescription drugs; physician education on evidence-based prescription practices of opioid analgesics; training of Emergency Medical Services (EMS) personnel in the use of naloxone for suspected opioid overdoses; and Good Samaritan laws to protect bystanders who administer naloxone in cases of suspected opioid overdoses $(9,10)$. In 2015, nearly 194 bills restricting provider's practices regarding opioid prescription were introduced in state legislatures, and of these, 61 were enacted into law (11). However, the effect of all these efforts on reducing opioid overdoses and deaths is not yet known.

Legislation has led to increased access to naloxone by emergency medical personnel and law enforcement. In the past 5 y, 46 states have passed laws enabling EMS personnel, including police officers, to administer naloxone in suspected cases of opioid overdose. Additionally, 46 states and the District of Columbia now have laws that provide immunity to medical professionals who prescribe or dispense naloxone and to laypersons who administer naloxone. In 2014, more than 150,000 laypersons received naloxone rescue training, with more than 26,000 reported overdose reversals (14).

Over the past several decades, naloxone has been safely used in prehospital and in-hospital settings for opioid reversal. Naloxone can be administered via the intramuscular (IM), subcutaneous, or intranasal (IN) routes. Its mechanism of action includes rapid onset when given by the intravenous (IV) route; its metabolism is primarily hepatic (15). Its effect on the reversal of opioid effects is dramatic, with clearing of the sensorium and restoration of spontaneous respirations within seconds in otherwise healthy patients; duration is 20 to $90 \mathrm{~min}$, allowing individuals to be brought to definitive medical care $(10,15)$.

Early delivery of intramuscular Epinephrine by nonmedical personnel in the prehospital setting at the onset of anaphylactic symptoms has been associated with reduced morbidity (12). It is therefore likely that providing naloxone and training in its use for caregivers of young adults and adolescents who misuse opioids would also be beneficial (13). A recent study identified demographic and drug-using patterns, and found that 
most opioid overdoses, including $86 \%$ of those by adolescents, occurred inside the home. Thus, opioid overdose response programs may more efficiently address the epidemic by both reducing the risk of overdose in the home through restriction of access to prescription opioids and targeting those who may be in the home at the time of an adolescent's overdose for training in the administration of naloxone (9).

Naloxone by IM and IN administration by bystanders has been shown to be safe and effective, with minimal training necessary $(13,16)$. In addition, administration of IN naloxone has been successfully used in an off-label manner in the prehospital setting (17). Provided there is no nasal trauma, epistaxis, or septal damage, no recent use of IN vasoconstrictive agents, and no concomitant significant hypotension that would preclude adequate intranasal absorption, IN naloxone can otherwise be administered (15). The Food and Drug Administration (FDA) has now approved an IN formulation of naloxone (Narcan nasal spray-Adapt) for emergency treatment of opioid overdose. It is the first nasal spray to be approved for this indication using a mucosal atomizer device. The drug also recently became available in an auto-injector formulation (Evzio) for IM or subcutaneous administration (18).

Naloxone for home use, whether an IM, subcutaneous or IN preparation, cannot simply be prescribed without a clear safety plan for its correct use by caregivers. Similar to safety planning for anaphylaxis events for parents and caregivers of children with food allergies, it is vital that caregivers of adolescents and young adults know how to use prescribed naloxone correctly and promptly, and then to seek timely medical care after its administration $(13,19)$.

The increasingly prevalent prescription of naloxone for caregivers and implementation of home safety planning for adolescents with opioid use disorders is a novel means to reduce injuries and deaths from opioid toxicity. Its addition to a multipronged strategy that includes restriction of prescription of opioids, safe disposal and storage of prescription opioids, and substance use treatment may be helpful to reverse the alarming trend of opioid overdoses among adolescents and young adults in the United States.

\section{YOUTH FIREARM INJURY}

Youth injured by firearms remains a topic of persistent national attention. According to the Centers for Disease Control and Prevention, homicide and suicide by firearms accounts for the second and fourth leading causes of death in the United States, respectively, for youth aged 15-24, with over 5,000 young people killed in 2014 alone (1). One study indicated that in comparison to other high-income countries, US gun homicide rates for 15-24-y olds was 49 times higher, and that firearm injuries disproportionately affect African-American and Latino males (20-22).

The scope of the problem of youth firearm injury has only recently become clearer, owing in part to database analyses. One example is the development of the National Violent Death Reporting System (NVDRS). NVDRS is a reporting system that is a repository for demographic and descriptive data on firearm injuries. Its sources include data extracted from death certificates, police reports, crime labs, and medical examiners $(21,23)$. This review will focus primarily on the development of the NVDRS and emphasize the development of research tools to track adolescent firearm injury by a public health approach. It is precisely this first step that represents a breakthrough in the field of firearm injury prevention research.

Significant advances in injury prevention serve as a reminder of the success of a public health approach to injury prevention (24). In order to create systems to study a public health problem like firearm injuries, researchers in the 1990s set out to create a "good data system that provides consistent and comparable detailed information across sites and over time" (25). In 1994, early steps were taken to create the data system that would be a repository for firearm injury data, modeled on similar systems for vehicular safety; however, these early efforts were not successful in securing funding from the federal government (26). Over the course of the next decade, many stakeholders from academic and nonprofit institutions were needed to pilot a data system for violent injury data that led Congress in 2002 to allocate funding for the development of the NVDRS (26).

Hemenway et al. demonstrated the utility and importance of this data system with multiple studies based on the growing NVDRS. In 2010, one study highlighted the differences between "self-inflicted" and "other-inflicted unintentional firearm deaths," thus demonstrating the impact of this data system on youth firearm injury research (23). This study showed that in other-inflicted unintentional shooting deaths, "the shooters were overwhelmingly young ( $81 \%$ under age 25$)$; the shooters were primarily friends (43\%) or family (47\%); and brothers were the most common family shooter" (23). Before the NVDRS, data on the shooter in unintentional gun deaths involving youth was not widely available, and this study was able to show that the majority of unintentional fatalities involving children were "other-inflicted," as opposed to self-inflicted (23). Notably, there were limitations to this study, namely only a third of States were providing data to the NVDRS at the time of the study, and these data do not provide the level of detail that a case-control study would provide. This work, however, did represent key findings that were the direct product of the first national database to track firearm injury, laying the groundwork for the necessary steps to create and implement effective, evidence-based, safety, and injury prevention measures. Similarly, through access to data in the NVDRS, detail on the number of suicides by firearm and homicides by firearm is now more widely available in terms of intentional injuries, and these two mechanisms account for the second and third leading causes of deaths in the age group 15-24 with over 9,000 deaths in the United States in 2014 alone (1).

Similarly, studies of hospitalization and emergency department visits for youth firearm injuries represent an approach to examining firearm injury at a higher level of detail than the NVDRS can provide. Studies by Leventhal et al. and Srivasan et al. serve as examples of research that addresses firearm injury by examining hospital administrative data on rates of hospitalization and visits to the emergency department/ambulatory sites and cost of firearm injury within the United States $(21,27)$. 


\section{Review | Dodington et al.}

Leventhal et al. performed a secondary analysis of the 2009 Kids' Inpatient Database, a nationally representative sample of discharge data from acute care hospitalizations. They found that in "2009 almost 7400 persons $<20$ y of age were hospitalized for firearm injuries" and that "the rates of hospitalizations were highest in 15- to 19-year-olds and in black males" (21). The major strength of this study was the large sample of hospitalizations for those younger than $20 \mathrm{y}$ of age. A key finding showed that males accounted for a vast majority of firearm injuries requiring hospitalization (89\%). In addition, firearm injuries accounted for a significant contribution to health disparities within this dataset, noting that among "US children and adolescents, males, blacks, and those receiving Medicaid were at the highest risk of firearm related hospitalizations. Furthermore, $70 \%$ of hospitalizations among black males could be attributed to assault" (21). Limitations of this study are similar to other studies of hospital administrative datasets: inherent difficulties in using medical billing codes to identify cases; and the risk of "double counting" individuals for sequelae of their injuries. Although the authors made efforts to mitigate these limitations, there is no current study tracking long-term outcomes of hospitalization due to firearm injury.

Srivasan et al. examined emergency department and ambulatory care visits in the United States between 2001-2010 using the National Hospital Ambulatory Medical Care Survey and ICD-9 codes to evaluate epidemiologic risk of injury through multivariate analysis. The study also demonstrated that African-American males, and those between 12-19 y old, are at the highest risk of firearm injury (27). Notably, their results demonstrated a rate of firearm injury that was $24 \%$ higher than previous estimates at that time $(1,27)$. Taken together, these two studies demonstrate the initial data needed to focus efforts on development of public health interventions on populations at the highest risk for firearm injury.

It should be noted that there are still significant gaps in researchers' ability to create, implement, and evaluate safety and injury prevention strategies around youth firearm injuries. Unlike other topics reviewed here, there is currently no definitive public health intervention that has demonstrated impact on outcomes in youth firearm injury, owing to the lack of clear outcomes research on this topic to date. However, the creation of the NVDRS and studies that track morbidity secondary to firearm injury represent a breakthrough in our ability to understand this critical public health problem that affects thousands of young people in the United States each year. At present, NVDRS has expanded to encompass data from 32 states, but at this time funding has not been allocated to incorporate data from the remaining states (28). Importantly, we must acknowledge the significant health disparities facing the population most affected by youth firearm injuries and dedicate the appropriate resources to understanding the longterm outcomes of these injuries.

\section{MOTOR VEHICLE SAFETY}

In the United States, motor vehicle crashes are one of the leading causes of unintentional morbidity and mortality for children and young adults between 1-24 y of age. Child safety restraint seats (CSRSs) are vital in the prevention of morbidity and mortality from motor vehicle crashes. Use of CSRSs reduces the risk of death in passenger cars by as much as $71 \%$ for infants and by $54 \%$ for toddlers $1-4$ y of age (29-32). For children 4-7 y of age, the use of booster seats has been found to reduce the injury risk by $45 \%$ compared to seat belt use alone $(30,32,33)$. Graduated Drivers Licensing (GDL) programs place restrictions on novice drivers under the age of 18 , and gradually eliminate license restrictions depending on the length of time one has held a license or learner's permit. Research performed on GDL programs suggests that the most stringent GDL programs are associated with reductions of 38 and $40 \%$ in fatal and nonfatal crashes, respectively, among 16-y-old drivers (34). Taken together, CSRSs and GDL lesgislation targets the injury mechanism that accounts for the most fatalities of children and young adults in the United States (Table 1). In this section, we will discuss both CSRS laws and GDL programs, and focus specifically on points where further legislative efforts could improve injury prevention.

A historical feature of child passenger safety legislation in the US has been the discrepancy between federal and state legislation requiring the use of CSRS and best practice recommendations from pediatric and automotive safety advocates. For example, in 1978, Tennessee became the first state to pass a Child Passenger Safety law requiring caregivers to place infants in CSRSs that met federal standards (35). Three years later, in 1981, a more stringent version of federal legislation (FMVSS 213-80) was passed to require CSRS manufacturers to include more stringent crash testing (including frontal crash testing) of rear-facing infant restraints, car beds, and forward-facing restraints for children under 50 pounds (36). Then, in 1985, there finally was legislation that mandated the use of such crash-tested CSRS in all 50 states, as well as Puerto Rico and the District of Columbia $(37,38)$.

Recent attempts to increase the use of child restraints have primarily come in the form of state legislation. The specific points of each law differ for each state, but the basic provisions include: (i) the age of the children affected (usually referring to all children under a specified number of years, e.g., $4 \mathrm{y}$ in Missouri and Tennessee, 3 y in Alabama); (ii) type of restraint required (federal standards); (iii) the conditions of seating (e.g., if in the front, the child must be in a safety seat; if in back, child must be in safety seat or car seat belt); (iv) person responsible for taking action (adult operator); and (v) the level of infraction for violation of the law (e.g., misdemeanor, fine, etc.) (38).

It has been well documented that laws mandating the correct use of booster seats is effective in preventing injuries and death (39). Concomitant levels of public awareness of a new restraint law also correlate with more children being restrained $(29,31)$. Child passenger restraint laws that increase the age required for car seat or booster seat also result in more children being restrained (29). In one study, there was a $17 \%$ decrease in death and serious injuries in five states that passed laws to increase the required age for CSRS use to 7 or 8 y of age (29). In addition, 
there was a threefold increase in the number of children who used car seats or booster seats (29).

Bae et al. examined the diffusion of child passenger safety laws in the United States over time, showing that there are continual changes to the laws that states create in response to motor vehicle safety recommendation (35). The group found that improved driver knowledge of their respective state child passenger restraint laws led to an increase in CSRS use, thus suggesting that public health awareness campaigns are effective in improving the desired behavior-use of a CSRS (35). However, at present, state laws often lag behind the safety advancements of the auto and child passenger safety industries; so even if parents and caregivers are cognizant of their state laws, they may not be incorporating best practices. This presents ongoing challenges for policymakers, given the need for recurring changes to existing child passenger safety laws and for caregivers, attempting to stay aware of constantly changing child passenger restraint laws and recommendations.

One example of a unifying recommendation that sets a standard for replication amongst child passenger legislation was the American Academy of Pediatrics 2011 recommendations. The key points of those recommendations included that children younger than 2 y old should ride rear-facing in a CSRS; children should be kept in a five-point harness system until they weigh at least 60 pounds and then use a booster seat until the age of eight and/or reaching a weight of 80 pounds (40). A concerted effort to make state legislation more uniform has not been successful to date, and might hold the key to a significant reduction in confusion for caregivers and policymakers, and improve overall use of CSRS.

Similarly, GDL laws would also likely benefit from standardization in best practices to reduce crashes by teen drivers and resultant injuries and deaths. GDL legislation was first developed in the 1990s, and Florida is credited with the first formal program in the United States (41). The drastic reduction in injuries for teens is likely because GDL laws eliminate multiple risk factors, such as number of passengers in a given vehicle, driving during more dangerous times of day, and general risktaking behaviors (42). Based on a risk calculator tool, one study by the Insurance Institute for Highway Safety indicated that if all 50 states enacted the most stringent GDL laws, the United States could save more than 500 lives a year (43). The hallmarks of the most stringent laws consists of "a minimum intermediate license age of 17," and "a minimum permit age of 16," and "during the intermediate stage, a night driving restriction starting at 8 p.m." as well as a "ban on all teen passengers" (43). Given the variation between states on these provisions, a unified set of recommendations adopted throughout all 50 states might have a significant impact on injury reduction. Given the burden of injuries from motor vehicle crashes for children and adolescents, it is imperative that further efforts be made so that all states incorporate best practices in their proposed CSRS and GDL legislation. Along with concomitant public awareness campaigns, parents and caregivers would then be able to provide the safest conditions for their children, whether as passengers or drivers, no matter the state in which they reside.

\section{CONCLUSIONS}

Taken together, these developments in injury prevention over the past several decades represent breakthroughs in an effort to reduce preventable injury from opioid toxicity, firearm injury, and motor vehicle crashes. Similar to the use of epinephrine injections by bystanders for anaphylaxis, the prescription of naloxone and implementation of safety planning will add to the multipronged strategy to reduce the alarming trend of opioid overdoses among adolescents and young adults in the United States. The creation of the NVDRS in 2002 has been vital in understanding the demographics and the significant health disparities facing the population most affected by youth firearm injuries and the further expansion and inclusion of epidemiological risk through national database research on firearm injury is critical to any future success in reducing injuries. Finally, through efforts to make CSRS laws and graduated driver license programs uniform and further expanded, we can begin to implement focused injury prevention programs and policies to reduce morbidity and mortality from motor vehicle crashes. Focused efforts to reduce unintentional injuries from opiate overdoses, firearms and motor vehicle crashes may produce a breakthrough in the field of injury prevention similar to that of the Poison Prevention Packaging Act.

\section{STATEMENT OF FINANCIAL SUPPORT}

No financial assistance was received in support of this review.

Disclosure: The authors have no conflicts of interest to declare.

\section{REFERENCES}

1. Centers for Disease Control and Prevention, National Center for Injury Prevention and Control. Web-based Injury Statistics Query and Reporting System (WISQARS: 2003), 2016. (http://www.cdc.gov/ncipc/wisqars).

2. Walton WW. An evaluation of the Poison Prevention Packaging Act. Pediatrics 1982;69:363-70.

3. Substance Abuse and Mental Health Services Administration. Prescription Drug Misuse and Abuse, 2016. (http://www.samhsa.gov/prescriptiondrug-misuse-abuse)

4. Frank D, Mateu-Gelabert P, Guarino H, et al. High risk and little knowledge: overdose experiences and knowledge among young adult nonmedical prescription opioid users. Int J Drug Policy 2015;26:84-91.

5. Substance Abuse and Mental Health Services Administration. Trends in Heroin Use in the United States: 2002 to 2013, 2016. (http://www.samhsa. gov/data/sites/default/files/report_1943/ShortReport-1943.html).

6. Centers for Disease Control and Prevention. Vital Signs: Overdoses of Prescription Opioid Pain Relievers --- United States, 1999-2008, 2016. (http:// www.cdc.gov/mmwr/preview/mmwrhtml/mm6043a4.htm).

7. Calcaterra S, Glanz J, Binswanger IA. National trends in pharmaceutical opioid related overdose deaths compared to other substance related overdose deaths: 1999-2009. Drug Alcohol Depend 2013;131:263-70.

8. Centers for Disease Control and Prevention. Increases in Drug and Opioid Overdose Deaths - United States, 2000-2014, 2016. (http://www.cdc.gov/ $\mathrm{mmwr} /$ preview/mmwrhtml/mm6450a3.htm?s_cid=mm6450a3_w).

9. Siegler A, Tuazon E, Bradley O'Brien D, Paone D. Unintentional opioid overdose deaths in New York City, 2005-2010: a place-based approach to reduce risk. Int J Drug Policy 2014;25:569-74.

10. Hawk KF, Vaca FE, D’Onofrio G. Reducing fatal opioid overdose: prevention, treatment and harm reduction strategies. Yale J Biol Med 2015;88: $235-45$.

11. National Conference of State Legislators. Injury Prevention Legislation Database, 2016. (http://www.ncsl.org/research/health/injury-preventionlegislation-database.aspx) 
12. Fleming JT, Clark S, Camargo CA Jr, Rudders SA. Early treatment of foodinduced anaphylaxis with epinephrine is associated with a lower risk of hospitalization. J Allergy Clin Immunol Pract 2015;3:57-62.

13. Jones JD, Roux P, Stancliff S, Matthews W, Comer SD. Brief overdose education can significantly increase accurate recognition of opioid overdose among heroin users. Int J Drug Policy 2014;25:166-70.

14. National Conference of State Legislatures. Drug Overdose Immunity and Good Samaritan Laws, 2016. (http://www.ncsl.org/research/civil-andcriminal-justice/drug-overdose-immunity-good-samaritan-laws.aspx).

15. Robinson A, Wermeling DP. Intranasal naloxone administration for treatment of opioid overdose. Am J Health Syst Pharm 2014;71: 2129-35.

16. Doe-Simkins M, Quinn E, Xuan Z, et al. Overdose rescues by trained and untrained participants and change in opioid use among substance-using participants in overdose education and naloxone distribution programs: a retrospective cohort study. BMC Public Health 2014;14:297.

17. Intranasal naloxone for treatment of opioid overdose. Med Lett Drugs Ther 2014; 56: 21-2.

18. The Medical Letter, Inc. Naloxone (Narcan) Nasal Spray for Opioid Overdose, 2016. (http://secure.medicalletter.org/).

19. Salter SM, Loh R, Sanfilippo FM, Clifford RM. Demonstration of epinephrine autoinjectors (EpiPen and Anapen) by pharmacists in a randomised, simulated patient assessment: acceptable, but room for improvement. Allergy Asthma Clin Immunol 2014;10:49.

20. Richardson EG, Hemenway D. Homicide, suicide, and unintentional firearm fatality: comparing the United States with other high-income countries, 2003. J Trauma 2011;70:238-43.

21. Leventhal JM, Gaither JR, Sege R. Hospitalizations due to firearm injuries in children and adolescents. Pediatrics 2014;133:219-25.

22. Grinshteyn E, Hemenway D. Violent death rates: the US compared with other high-income OECD countries, 2010. Am J Med 2016;129:266-73.

23. Hemenway D, Barber C, Miller M. Unintentional firearm deaths: a comparison of other-inflicted and self-inflicted shootings. Accid Anal Prev 2010;42:1184-8.

24. Mozaffarian D, Hemenway D, Ludwig DS. Curbing gun violence: lessons from public health successes. JAMA 2013;309:551-2.

25. Hemenway D, Miller M. Public health approach to the prevention of gun violence. N Engl J Med 2013;368:2033-5.

26. Hemenway D, Barber CW, Gallagher SS, Azrael DR. Creating a National Violent Death Reporting System: a successful beginning. Am J Prev Med 2009;37:68-71.

27. Srinivasan S, Mannix R, Lee LK. Epidemiology of paediatric firearm injuries in the USA, 2001-2010. Arch Dis Child 2014;99:331-5.
28. Rubin R. Tale of 2 agencies: CDC avoids gun violence research but NIH funds it. JAMA 2016;315:1689-91.

29. Centers for Disease Control and Prevention. CDC VitalSigns - Child Passenger Safety, 2014. (http://www.cdc.gov/vitalsigns/childpassengersafety).

30. Centers for Disease Control and Prevention. Child Passenger Safety: Get the Facts | Motor Vehicle Safety, 2016. (http://www.cdc.gov/MotorVehicleSafety/Child_Passenger_Safety/CPS-Factsheet.html).

31. National Highway Traffic Safety Administration. NCSA Publications \& Data Requests, 2016. (http://www-nrd.nhtsa.dot.gov/CATS/listpublications.aspx? Id=A\&ShowBy=DocType).

32. Sauber-Schatz EK, West BA, Bergen G; Centers for Disease Control and Prevention (CDC). Vital signs: restraint use and motor vehicle occupant death rates among children aged 0-12 years - United States, 2002-2011. MMWR Morb Mortal Wkly Rep 2014;63:113-8.

33. Durbin DR, Runge J, Mackay M, et al. Booster seats for children: closing the gap between science and public policy in the United States. Traffic Inj Prev 2003;4:5-8.

34. Baker S, Chen L, Li G. Nationwide review of graduated driver licensing. AAA Foundation for Traffic Safety, 2007. (http://www.aaafoundation.org/ pdf/NationwideReviewOfGDL.pdf).

35. Bae JY, Anderson E, Silver D, Macinko J. Child passenger safety laws in the United States, 1978-2010: policy diffusion in the absence of strong federal intervention. Soc Sci Med 2014;100:30-7.

36. National Highway Traffic Safety Administration. Federal Motor Vehicle Safety Standards and Regulations, 2016. (http://www.nhtsa.gov/cars/rules/ import/FMVSS/index.html - SN213).

37. Gardner HG, Baum CR, Dowd MD, et al. Policy statement-child passenger safety. Pediatrics 2011; 127: 788-93.

38. Governors Highway Safety Association. State Child Passenger Safety Laws, 2014. (http://www.ghsa.org/html/stateinfo/laws/childsafety_laws.html).

39. Mannix R, Fleegler E, Meehan WP 3rd, et al. Booster seat laws and fatalities in children 4 to 7 years of age. Pediatrics 2012;130:996-1002.

40. Durbin DR; Committee on Injury, Violence, and Poison Prevention. Child passenger safety. Pediatrics 2011;127:e1050-66.

41. McCartt AT, Teoh ER. Strengthening driver licensing systems for teenaged drivers. JAMA 2011;306:1142-3.

42. Simons-Morton B, Lerner N, Singer J. The observed effects of teenage passengers on the risky driving behavior of teenage drivers. Accid Anal Prev 2005;37:973-82.

43. Insurance Institute for Highway Safety. States could sharply reduce teen crash deaths by strengthening graduated driver licensing laws, 2012 (http://www.iihs.org/iihs/news/desktopnews/states-could-sharply-reduceteen-crash-deaths-by-strengthening-graduated-driver-licensing-laws). 